Comput. Mater. Sci., revised manuscript

\title{
Evolution of internal strain in austenite phase during thermally induced martensitic phase transformation in NiTi shape memory alloys
}

\author{
Sourav Gur ${ }^{1}$, Venkateswara Rao Manga ${ }^{2}$, Stefan Bringuier ${ }^{2}$, Krishna Muralidharan ${ }^{2}$ and \\ George N. Frantziskonis ${ }^{1,2, *}$ \\ ${ }^{1}$ Civil Engineering and Engineering Mechanics, University of Arizona, Tucson, AZ 85721 USA \\ ${ }^{2}$ Materials Science and Engineering, University of Arizona, Tucson, AZ 85721 USA \\ * Corresponding author Email: frantzis@email.arizona.edu (G. N. Frantziskonis)
}

\begin{abstract}
New insight into the temperature dependent evolution of internal strain in the austenite phase during the martensitic phase transformation in NiTi shape memory alloys is provided via classical molecular dynamics simulations that employ well-established interatomic potentials for NiTi. It is shown, for the first time, that the developed strain tensor in the austenite phase is tetragonal in nature, with exponential temperature-dependence. Equally importantly, it is found that the developed internal strain (parallel to the habit plane) in the austenite varies linearly with the evolving martensite phase fraction. Interestingly, the Richard's equation is found to describe the temperature dependence of the martensite phase fraction as well as the internal strain components parallel to the habit plane in the austenite phase. An analysis of the temperature dependent phonon dispersion of strained austenite revealed the competition between phonon softening of the $\mathrm{TA}_{2}$ branch and internal strain that leads to stabilization of the austenite phase in the two phase regime.
\end{abstract}

Keywords: NiTi shape memory alloy, transformation-induced strain, Richard's equation and phonon instability.

\section{Introduction}

Shape memory alloys (SMAs) recover inelastic or residual strain through phase transformation upon heating; this is known as the shape memory effect (SME). SMAs such as NiTi can also recover large inelastic or residual strains (up to $8 \%$, above the martensite start temperature) through reverse phase transformation upon unloading; this is known as superelasticity. Due to such properties NiTi has found applications in many areas, and at scales ranging from nanometer to macro continuum level [1-3]. The SME, associated with the thermally induced martensitic (i.e. austenite (B2) to martensite (B19 $\square$ ) and vice versa) phase transformation in NiTi SMA has been exploited widely [1,4-7]. It is observed that, the evolution and growth rate kinetics of different phases in NiTi SMAs at the continuum level differs from that at the nanometer scale, mainly because of the vast amount of free surfaces at the nanometer scale [6-8]. The thermally 
induced martensite phase transformation process along length scales is studied in [6], i.e. information on the evolution of $\mathrm{B} 2$ and different $\mathrm{B} 19 \square$ variants (due to the temperature) is tracked from atomic to mesoscale to continuum scale. Other experimental studies [9-11] reported that, size effects on the evolution of compound twin martensite variants in nano crystalline NiTi, are due to the length scale or size dependent strain energy barrier. Also, the atomistic study [12-14] found that, in coarse-grained NiTi samples, type I and II twins are more noticeable than compound twins. This is due to the deformation compatibility requirements for the habit planes (i.e. interface of austenite and different martensite variants). Even after all these extensive studies, the evolution and stability of different phases during the phase transformation process in NiTi SMA at the atomic or nanometer scale is not well-understood.

It is well established that the transformation from austenite to martensite is displacive in nature and occurs via an underlying phonon instability due to the softening of a specific transverse acoustic $\left(\mathrm{TA}_{2}\right)$ branch in its phonon dispersion [1,15-17]. During the process of displacive transformation, NiTi B2 crystals experience shear distortion due to the combination of shear along the basal (on (110) [ [ $\left.\begin{array}{lll}1 & 1 & 0\end{array}\right]$ ) and a non-basal (on (001) [ $\left.\begin{array}{lll}1 & 1 & 0\end{array}\right]$ ) planes, and transform into the monoclinic B19] (martensite) phase $[18,19]$. Further, it is also known that the phase transformation occurs over a range of temperature with the transformation temperature continuously changing during cooling $[1,20]$. Specifically, below the martensite start temperature $\left(\mathrm{M}_{\mathrm{s}}\right)$, the formation of martensite leads to the development of transformation induced internal strain, which in turn resists further transformation of the austenite. As a result, the remaining austenite exhibits a lower transformation temperature, i.e. requires continuous cooling in order to acquire an increased driving force to advance further the transformation process. In terms of the underlying thermodynamics of the martensitic phase transformation process, the resistance caused by the transformation induced strain is quantified as an elastic strain energy [21,22].

To the authors' best knowledge, the exact nature and magnitude of these transformation induced strains in the austenite phase is unknown. Furthermore, experimental determination of the localized lattice strains at the nanoscale is not trivial. The localized strain measurements employing techniques such as convergent electron beam diffraction (CBED) to get the 3D strain tensors are highly cumbersome [23], while the diffraction measurements carried over multiple grains yield averaged strains that may not reveal the underlying non-hydrostatic nature of the developing strains [24]. Several studies based on crystallographic theory for bulk NiTi SMA [25-28], provide information on (a) the nature and the magnitude of the developed internal strain in the monoclinic B19 $\square$ variants (due to the phase transformation process); and (b) the nature and type of interfaces that grow (i.e. habit planes) between B2 and B19 $\square$ variants. A recent computational work at the mesoscale (employing dislocation theory) [29] studies the interaction between temperature and plastic deformation on the evolution of martensite phase fraction and provides the temperature dependency of the developed average internal strain. However, nothing specifically is mentioned about the exact nature and the magnitude of individual components of the strain tensor. Therefore, in addition to the lack of understanding about the nature of the transformation induced internal strains in the austenite phase at the two-phase temperature 
region, there are other outstanding questions regarding the martensitic phase transformation in NiTi alloys, namely: (a) how does the underlying internal strain tensor in austenite vary as a function of temperature? (b) how are the temperature, martensite phase fraction and internal strain interrelated? and (c) how does the coupled effect of temperature and evolving internal strain stabilizes the $\mathrm{TA}_{2}$ branch of the austenite phase within the two-phase temperature regime?

As a first, yet effective level of study, the aforementioned questions are addressed herein using classical molecular dynamics (MD) simulations that employ well-established interatomic potentials for NiTi SMAs. In particular, the transformation-induced strain tensor in the austenite phase as a function of temperature between the martensite start $\left(\mathrm{M}_{\mathrm{s}}\right)$ and martensite finish $\left(\mathrm{M}_{\mathrm{f}}\right)$ temperatures is examined and characterized. The martensite phase fraction $(\square)$ and the internal strain tensor $\left(\varepsilon_{i j}\right)$ in the austenite phase are described as a function of temperature within the twophase (austenite + martensite) region. By employing fluctuation dissipation method, the details of how the developed internal strains stabilize the otherwise unstable $\mathrm{TA}_{2}$ branch in the phonon dispersion of the austenite phase are demonstrated. Interestingly, the obtained $\varepsilon_{\mathrm{ij}}$ - $\mathrm{T}$ relations are found to obey the same Richards equation [30,31] that describes the $\square$-T relations and hence leading to an accurate phenomenological relationship, between the phase fractions, temperature, and the developed internal strains.

\section{Methods}

This section provides information on the MD simulation process in LAMMPS [32,33] and on the characterization techniques used to separate the spatial domain of austenite from that of martensite phase. A brief description of the simulation methods is given since they are wellestablished and described in details in the literature [5-8,13,14,34-38].

\subsection{Molecular dynamics (MD) simulations}

MD simulations were performed within the LAMMPS [32,33] software framework, employing a previously proven well effective interatomic potential [5-8,13,14,38,37,34-36] for capturing the phase transformation in the NiTi SMA. Here, simulations are performed in all three spatial dimensions (3D). A well-established many-body Finnis-Sinclair potential is used for representing the interatomic interactions which was initially developed by Lai and Liu [39] based on the embedded-atom method (EAM) considering a second-moment approximation of tight-binding theory (TBSMA). This potential was modified by Zhong et al $[13,14]$ by adding a smooth cutoff function to avoid problems associated with an abrupt cutoff interaction radius and thus is used for the present study. Zhong et al [13,14] employed this potential to investigate the microstructure evolution of NiTi and found good agreement (i) with the phase transformation temperatures reported in the experimental literatures (agreement within $20 \mathrm{~K}$ ) [40] and (ii) in terms of martensite (B19 $\square$ ) twins structure (type-I, type-II and (001) or (010) compound twins) $[10,27]$ and its associated transformation matrices (i.e. from B2 phase to different B19 $\square$ variants) [25-27] based on the experimental studies [10,11] as well as crystallographic theory [25-27]. Additionally, this potential emulates relevant experimental and ab initio data such as lattice 
parameters of the $\mathrm{B} 2, \mathrm{~B} 19 \square$ and $\mathrm{BCO}$ phases $[25,41]$ and the elasticity stiffness tensor components of the $\mathrm{B} 2$ phase $[42,43]$.

\subsubsection{Interatomic potential}

According to the potential mentioned above, the total energy of the system is expressed as

$$
E_{t}=\sum_{i}\left\{\sum_{i \neq j} E\left(r_{i j}\right)-\sqrt{\sum_{i \neq j} F\left(r_{i j}\right)}\right\}
$$

In Eq. (1), the first term in the right hand side indicates a pairwise interaction energy while the second term represents many-body effects, considering second moment approximation for the electron density. Recently, the many-body interaction part of Lai and Liu potential has been modified by Zhong et al [13,14]. In this modified potential [13], a smooth cutoff behavior is considered to avoid the diverging forces during simulations [13]. Such modification ensures the continuity of the second term in Eq. (1) and its first derivative at cut off radius $\left(r_{c}\right)$ and hence provides smooth behavior in energies and interatomic forces, molecular statics and dynamics simulation. Different parameters of this modified interatomic potential are determined by fitting the properties (obtained from first principles calculations) of the $\mathrm{B} 2$ phase at $0 \mathrm{~K}$, with the potential cut off radius $r_{c}=4.2 \AA$. The terms in Eq. (1) are expressed as

$$
\begin{aligned}
& E\left(r_{i j}\right)=A_{\alpha \beta} \exp \left[-p_{\alpha \beta}\left(\frac{r_{i j}}{d_{\alpha \beta}}-1\right)\right] \\
& F\left(r_{i j}\right)=\left\{\begin{array}{cc}
\sum_{i \neq j} \xi_{\alpha \beta}^{2} \exp \left[-2 q_{\alpha \beta}\left(\frac{r_{i j}}{d_{\alpha \beta}}-1\right)\right] & \text { for } r_{i j} \leq r_{1} \\
c_{0, \alpha \beta}+c_{1, \alpha \beta}\left(r_{i j}-r_{1}\right) & \text { for } r_{1} \leq r_{i j} \leq r_{c} \\
+c_{2, \alpha \beta}\left(r_{i j}-r_{1}\right)^{2}+c_{3, \alpha \beta}\left(r_{i j}-r_{1}\right)^{3} &
\end{array}\right.
\end{aligned}
$$

where $i$ and $j$ denote atoms, $\alpha, \beta$ denote the type of atoms ( $\mathrm{Ni}$ or $\mathrm{Ti}$ ) and $r_{1}=4.0 \AA$. A detailed description of the parameters involved in this interatomic potential is provided in Zhong et al [13].

\subsubsection{Simulation process}

In order to study the austenite (B2) to martensite (B19 procedure is adopted. In all cases the bulk NiTi sample is initially pristine austenite B2 NiTi with the crystallographic orientation [001], [110] and [1 11 0]. B2 supercells with periodic boundary conditions are constructed in a fashion consistent with the work of Zhong et al [13,14], and it is seen that relevant quantities such as lattice parameters, phase fractions and strains are wellconverged at a supercell cell size of $75 \AA \times 75 \AA \times 200 \AA$ (86832 atoms), as will be shown subsequently. Furthermore, simulation results are verified with other crystallographic orientation i.e. for [111] orientations, with size 76.5 $\AA \quad 76.5 \AA \quad \times \quad 210 \AA$ (87480 atoms). 
At first, the system under study is geometrically optimized using the stress controlled conjugate gradient energy minimization method. MD simulations are carried out at temperatures in the range of 50-700 K with an interval of $10 \mathrm{~K}$ within the phase transformation temperature regime, i.e. $\mathrm{T}=250-450 \mathrm{~K}$, and at a $50 \mathrm{~K}$ interval for the remaining temperature ranges. Consideration of such large temperature range is important for accurate estimation of the martensite start and finish temperatures (i.e. $M_{s}$ and $M_{f}$ ) and phase fraction evolution, as detailed subsequently. At each temperature, the systems are initially subjected to NPT (i.e. constant pressure) conditions for 360 ps where the shape and size of the simulation boxes are allowed to change to ensure that both pressure as well as the individual components of the symmetric stress tensor are set to zero. This is followed by constant volume (NVT) conditions for an additional 240 ps. In each case of NPT or NVT stabilization, the adopted time step is $\Delta t=i \quad 0.0005$ ps. During simulations, the temperature (and pressure) is controlled by a Nose-Hoover thermostat (and barostat). In order to remove the effects of thermal fluctuations while analyzing structural characteristics (carried out during the NVT stage), the atomic coordinates are averaged over 20 ps. It is verified that the systems are well-equilibrated and converged during both NPT and NVT simulations. The fluctuations (with respect to the mean values) in total energy and temperature are smaller than $0.001 \mathrm{eV} /$ atom and $0.50 \mathrm{~K}$, respectively.

\subsection{Structural characterization and analysis}

To distinguish austenite B2 phase from martensite B19 $\square$ phase and its variants, the bond length based order parameter proposed by Mutter and Nielaba [8,35] is adopted. According to this order parameter, a pristine $\mathrm{B}^{\prime} 9^{\prime}$ structure has a value of $1.0 \pm 0.05$, where the uncertainty corresponds to fluctuations in the bond distances due to a combination of internal strain, interfacial effects and thermal expansion. It can be expressed as

$$
\chi\left(d_{0}, d_{1}\right)=\frac{\left[d_{0}\left\{d^{(B 2)}+d_{1}^{\left(B 19^{\prime}\right)}\right\}\right]-\left[d_{1}\left\{d^{(B 2)}+d_{0}^{\left(B 19^{\prime}\right)}\right\}\right]}{\left[d^{(B 2)}\left\{d_{0}^{\left(B 19^{\prime}\right)}-d_{1}^{\left(B 19^{\prime}\right)}\right\}\right]}
$$

where different bond length parameter of pure austenite phase and martensite phase are $d^{(B 2)}=2.62 \AA, d_{0}^{\left(B 19^{\prime}\right)}=2.46 \AA \wedge d_{1}^{\left(B 19^{\prime}\right)}=2.65 \AA$. The value of this order parameter becomes +1.0

for the martensite B19' phase when $d_{0}=d_{0}^{\left(B 19^{\prime}\right)}$ and $d_{1}=d_{1}^{\left(B 19^{\prime}\right)}$. For the austenite B2 phase $d_{0}=d_{1}=d^{(B 2)}$ and thus the value of this order parameter becomes -1.0 .

Structural analysis is done using atomic configurations obtained after averaging over the past 20 ps in the stabilized regime of the NVT simulations. As mentioned before, the lattice parameters of B2 and B19 $\square$ phases change because of the linear thermal expansion or contraction $(\alpha \Delta T)$, and because of the internal strains $\left(\varepsilon^{i}\right)$ that develop during the phase transformation process. Thus, instead of showing distinct values (like delta function) at +1.0 (for B19 $\square$ phase) or -1.0 
(for B2 phase), this bond length based order parameter for B19 and B2 phase exhibits a narrow Gaussian distribution around +1.0 and -1.0 , respectively [5-8,35]. It has been observed that this order parameter shows Gaussian distribution around a mean of $\mu_{x}= \pm\left(1 \pm \alpha \Delta T \pm \varepsilon^{i}\right)$, and with

standard deviation of $\sigma_{x}=\sqrt{(\alpha \Delta T)^{2}+\left(\varepsilon^{i}\right)^{2}}$. Based on the data from all performed simulations, it is observed that the order parameters for all B19 $\mathrm{C}$ phase atoms are greater than +0.95 , and for all B2 phase atoms are less than -0.95 . Thus, in this study, atoms with order parameter greater than +0.95 correspond to martensite B19 $\square$ phase and those less than -0.95 to austenite B2 phase. At every temperature, the martensite phase fraction is calculated based on the intensity and spread of the Gaussian curves. First, the area under each of the Gaussian curves and the total area under both Gaussian curves (i.e. total number of atoms) are calculated. The mean value of the martensite phase fraction is determined by taking the ratio of the area of Gaussian curve of the martensite (B19]) phase with respect to the total number of atoms in the simulation cell. The spread in the Gaussian curve of the martensite (B19]) phase provides a measure of uncertainty in the martensite phase fraction evolution. A more detailed description of the martensite phase fraction calculation based on this order parameter is provided in Mutter and Nielaba $[8,35]$ and in other relevant studies by the authors [5-7].

Once the spatial domains of the austenite (B2) phases in the simulation cell are located, the lattice parameters (i.e., bond lengths a, b and c, between the $\mathrm{Ni}-\mathrm{Ni}$ and/ or Ti-Ti atoms) are determined. It is also consistently observed that the bond angle between $\mathrm{Ni}-\mathrm{Ni}-\mathrm{Ni}$ and $\mathrm{Ti}-\mathrm{Ti}-\mathrm{Ti}$ atoms are approximately $90^{\circ}$. To accommodate for the inconsistency between B2 and B19 lattice parameters, (both bond length and bond angle), a few atomic layers near the B2-B19] interface are distorted. Thus, to eliminate any disorder or inconsistency in the measurement of lattice parameters in the B2 phase and thus in the developed internal strain, a couple of atomic layers in the B2 phase adjacent to the B2-B19 $\square$ interface are neglected.

\subsection{Phonon band structure simulations}

The anharmonic phonon band structure calculations are carried out using the fluctuationdissipation theory implemented in LAMMPS [32,33] software, employing the same interatomic potential. This technique determines the dynamical matrix from MD simulations employing the Green's function approach, enabling the calculations of the phonon eigenvalues and eigenvectors within the first Brillouin zone. An NVT ensemble with a supercell size of $8 \times 8 \times 8$ (1024 atoms) was employed to obtain the instantaneous and the averaged atomic coordinates of each atom to determine the coefficients of the dynamical matrix. An examination of size effects on the ensuing phonon dispersion showed that the employed size was sufficient. The simulations were run for a total time of $10 \mathrm{~ns}$ and the averaging on the atomic coordinates was performed over the last 50 ps. It is worth mentioning that the employed potential provides a qualitative description of the various acoustic and optic phonon branches. However, there are some deviations between the phonon dispersion curves as predicted by the employed potential and by first-principles methods [44] as will be revealed subsequently. 


\section{Results and discussion}

\subsection{Simulation cell size and microstructure in single and two phase region}

Figure 1 shows the distribution of the order parameter and the effect of simulation cell size on the phase fraction evolution. Figure 1a shows the bond length based order parameter for B2 and B19' phases at $370 \mathrm{~K}$, which exhibits a Gaussian distribution around -1 and +1 , respectively. The distribution of the order parameter arises due to the internal strains $(\varepsilon)$ and effect of temperature on B2 and B19 phases. The mean value and the standard deviation of the bond length based order parameter can be expressed as $\mu_{\chi}= \pm\left(1 \pm \alpha \Delta T \pm \varepsilon^{i}\right)$ and $\sigma_{\chi}=\sqrt{(\alpha \Delta T)^{2}+\left(\varepsilon^{i}\right)^{2}}$, respectively, where $\alpha$ is the linear thermal coefficient of expansion for a given phase. The convergence of temperature dependence of martensite phase fraction with respect to the supercell size is shown in Fig. 1 b. It is observed that with increasing simulation cell size, the temperature dependent evolution of martensite phase fraction and the transformation temperatures (martensite start and finish) converge (the difference between size-II and III results is approximately $6.5 \%$ ) and finally stabilize at the largest size i.e. size-III (75 $\AA \quad 75 \AA \quad \times \quad 200 \AA$ : 86832 atoms). Thus, simulation results for size-III are only shown, and results for other sizes are shown in the supplementary material. It is noted that all simulations were performed under the same conditions.
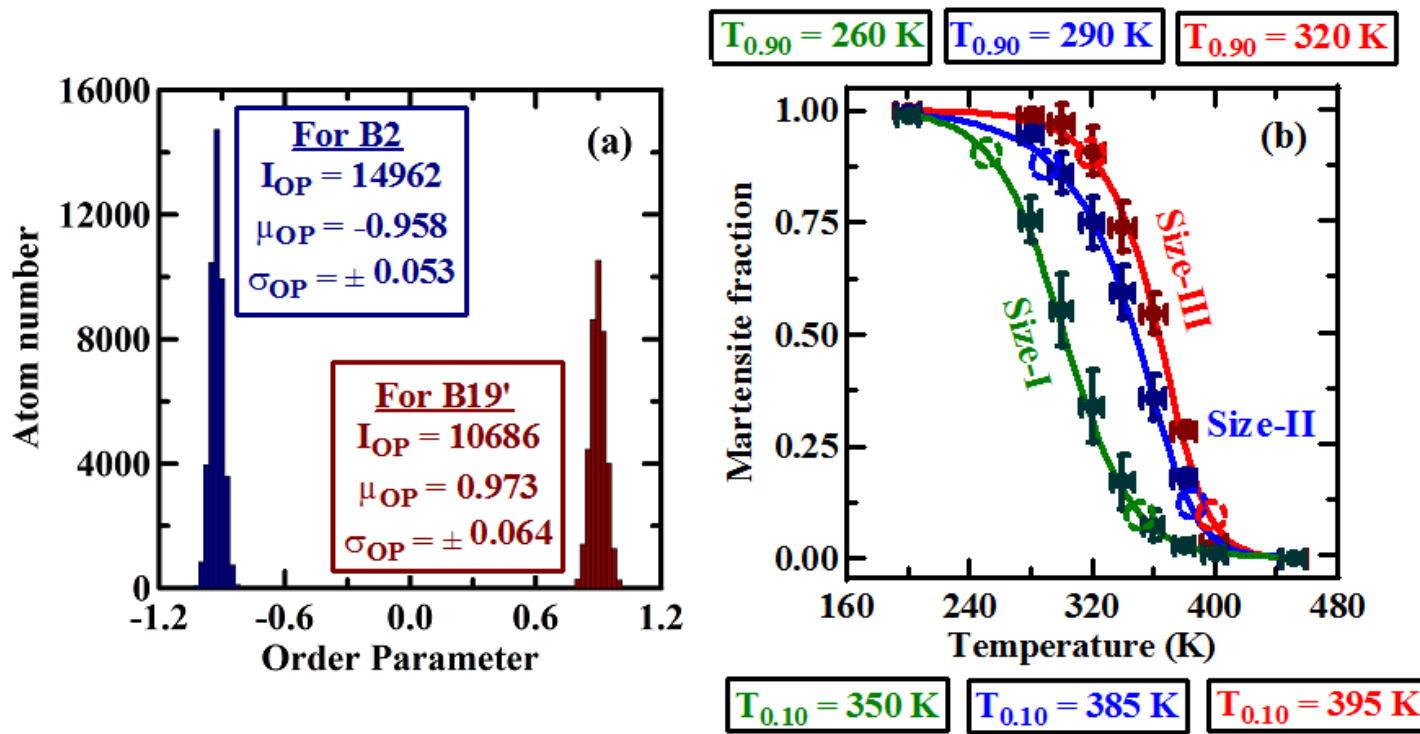

Figure-1: (a) Histogram of number of atoms with respect to the order parameter at $\mathrm{T}=370 \mathrm{~K}$. Gaussian distribution for B2 and B19', around -1 and +1 are shown, respectively. (b) Variation of martensite phase fraction as a function of temperature for NiTi supercells (of [001] orientation) of different sizes: size-I (25 $\times \quad 25 \AA \quad \times \quad 200 \AA: 9648$ atoms $)$, size-II $(50 \AA \times 50 \AA$ $\times 200 \AA$ : 32432 atoms) and size-III (75 $\AA \quad 75 \AA \quad \times \quad 200 \AA$ : 86832 atoms). Here, $\mathrm{T}_{0.90}$ and $\mathrm{T}_{0.10}$ represents temperature corresponding to the $90 \%$ and $10 \%$ martensite phase fractions, respectively. Note that below $10 \%$ and beyond $90 \%$ martensite phase fraction, the standard 
deviations on the fractions of the minor phase are of the order of the estimated phase fraction itself.

Figure 2 shows the microstructure of NiTi at three different temperatures, 250, 370 and $450 \mathrm{~K}$. The microstructure at $250 \mathrm{~K}$, as shown in Fig. 2a, shows a single-phase martensite with (001) compound twins, and this is in good agreement with earlier work reported by Zhong et al [13]. As shown in Fig. 2b, at $370 \mathrm{~K}$ the microstructure shows a two-phase mixture wherein the (001) plane forms the interface (i.e. the habit plane) between the austenite and martensite phases, as also reported by Zhong et al [13]. Further, the formation of the (001) habit plane is attributed to a combination of shear along the basal (on (110) [ [ $\left.\begin{array}{lll}1 & 1 & 0\end{array}\right]$ ) plane and a non-basal (on (001) [1 1 0]) plane in austenite, in agreement with what is reported by Otsuka and Ren [18]. The predicted microstructure is independent of the crystallographic orientations of the chosen supercell in terms of the phase fractions and morphology of phases. Simulations performed by supercells with a different orientation ([10 1 i ], [01 1 ] ] and [111] as a, b, and c axes) have also predicted similar results (see Supplementary material).

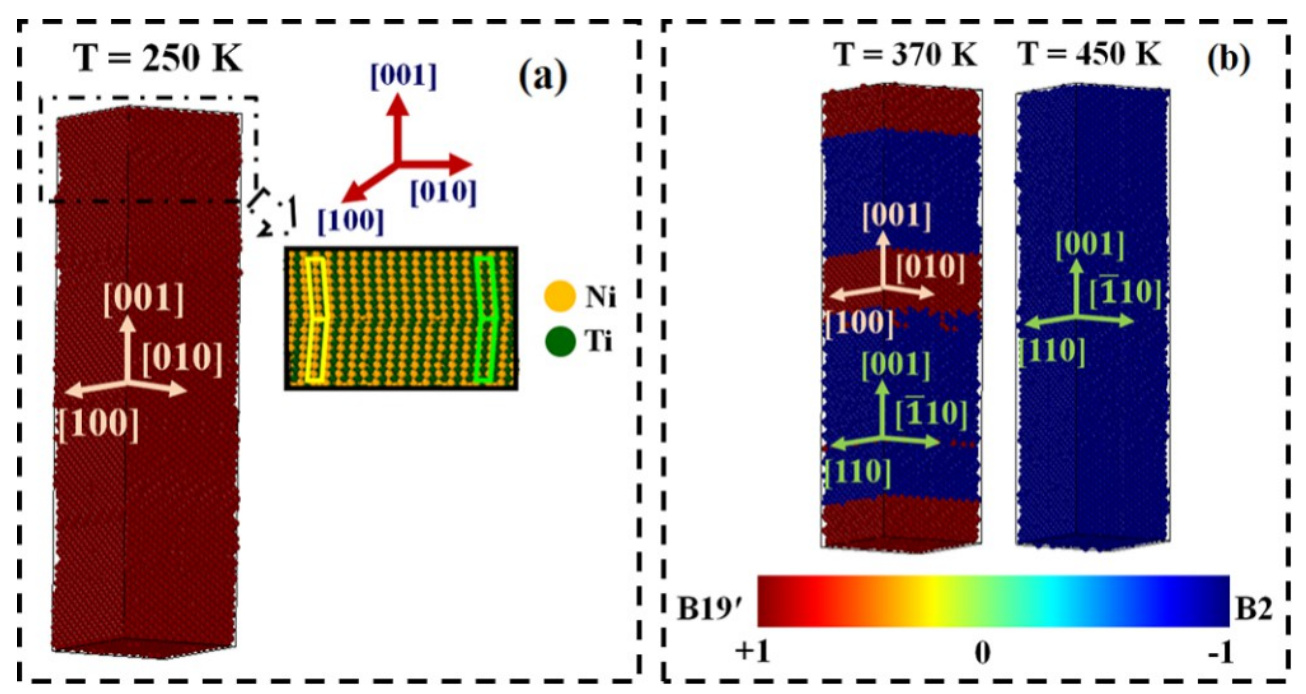

Figure-2: NiTi simulation cells of size $75 \AA \times 75 \AA \times 200 \AA$, after stabilization at different temperatures (a) a single phase martensite microstructure with (001) compound twins at $\mathrm{T}=250 \mathrm{~K}$; (b) microstructure at $\mathrm{T}=370 \mathrm{~K}$ and $450 \mathrm{~K}$, depicting the martensite (in red) and austenite (in blue) phase fractions. The crystallographic orientations of B2 and B19 $\square$ are marked by arrows in the respective phase regions.

\subsection{Lattice parameters and developed internal strain in austenite phase}

The austenite phase in the two phase-regions was analyzed through bond-length base order parameter and results are shown in Figure 3. Here, results (lattice parameters and internal strain in B2 phase) for size-III is only shown and for other sizes they are provided in the supplementary material. This analysis revealed that in contrast to the high-temperature cubic phase, the austenite phase in the two-phase region possessed tetragonal symmetry $(\mathrm{a}=\mathrm{b} \neq \mathrm{c})$, with the variation in the lattice parameters as a function of temperature as shown in Fig. 3a. This change in the 
austenite crystal structure indicates a tetragonal nature of the internal strain tensor that stabilizes the 'strained' austenite phase in the two-phase region.

In order to examine the transformation strains and their temperature dependence, the temperature dependent lattice parameters of the strained tetragonal austenite phase and the metastable cubic austenite phase at temperatures where the tetragonal austenite and martensite phases co-exist (i.e. below $\mathrm{M}_{\mathrm{s}}$ ) are compared. Specifically, the lattice parameters of the single-phase cubic austenite at high temperatures is extrapolated to the temperature(s) of interest. Such an extrapolation can be supported by the fact that (i) in experiments the $\mathrm{M}_{\mathrm{s}}$ can be lowered by employing different cooling rates $[45,46]$, and (ii) the lattice parameter of austenite varies linearly with temperature. The calculated strains for the tetragonally deformed austenite revealed that in the two-phase region the austenite phase experiences more compressive strain along the [100] and [010] directions (i.e. along a, b) than along the [001] direction (i.e. along c) with the transformationinduced tetragonal strain tensor that stabilizes the austenite phase below $M_{s}$ given by,

$$
\left(\begin{array}{ccc}
\varepsilon_{1} & 0 & 0 \\
0 & \varepsilon_{1} & 0 \\
0 & 0 & \varepsilon_{2}
\end{array}\right) .
$$

Figure $3 \mathrm{~b}$ shows the temperature dependence of the obtained strains $\left(\varepsilon_{1}, \varepsilon_{2}\right)$ in austenite in the two-phase region. The orientation of the strain tensor in the austenite phase with respect to the habit plane can also be seen in the Fig. 3b inset. As evident from the figure, within the austenite phase, the strain components in the directions parallel to the habit plane, i.e. along [100] and [010] directions are the same, while the strain along the direction perpendicular to the habit plane, i.e. along [001], is approximately $1 / 3$ of the strain along the [100] and [010] directions.
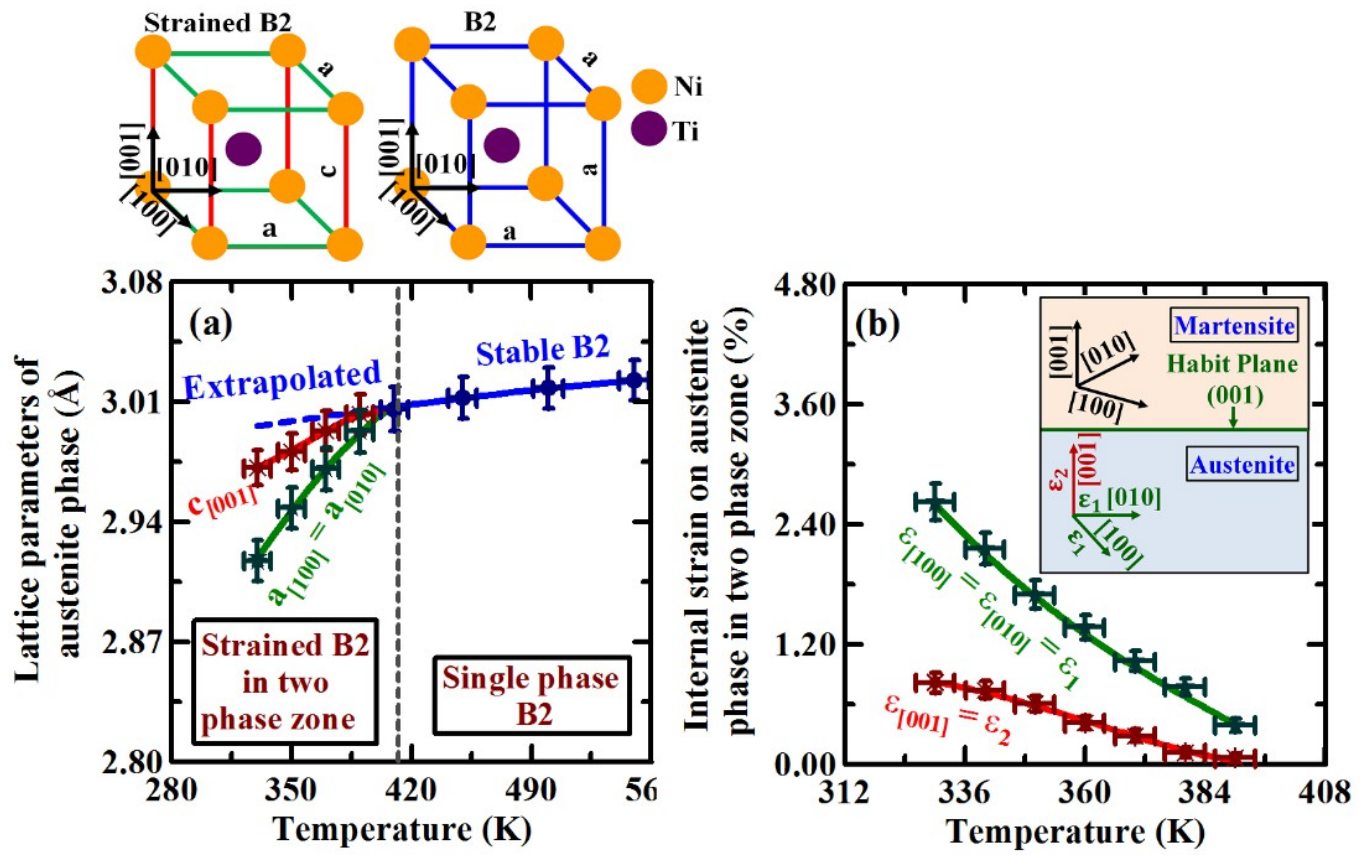
Figure-3: (a) Lattice parameters of stable and metastable austenite phases as a function of temperature. (b) Variation of tetragonal internal strain components in austenite in the two-phase region as a function of temperature.

\subsection{Relation among temperature, martensite phase fraction and internal strain in austenite}

As the next step, the temperature dependence of the strain tensor in the austenite phase is analyzed in relation to the evolving phase fraction of the martensite phase. Figure 4 shows the martensite phase fraction and normalized principal strains in the [100] or [010] direction (parallel to the (001) habit plane) as a function of temperature, for martensite phase fractions in the range of $10 \%$ to $90 \%$. Below $10 \%$ and beyond $90 \%$ martensite phase fraction, it was seen that the standard deviations associated with the estimated fractions of the minor phase were of the order of the phase fraction itself. As shown in Fig. 4, the temperature dependence of the martensite phase fraction is well described by the Richards equation [5-7,30,31], i.e.

$$
\xi(T)=1-\left[1+\exp \left\{-g \cdot v\left(T-T_{m}\right)\right\}\right]^{(-1 / v)}=\frac{\varepsilon_{1}(T)}{\varepsilon_{\max }}
$$

where, $\xi$ denotes the martensite phase fraction and $T_{m}$ the temperature corresponding to the maximum transformation rate, while $g$ and $v$ are the fit parameters which dictate the transformation rate. Further $\varepsilon_{1}$ and $\varepsilon_{\max }$ are the transformation induced internal strain at any temperature, along the [100] or [010] direction and its maximum value, respectively.

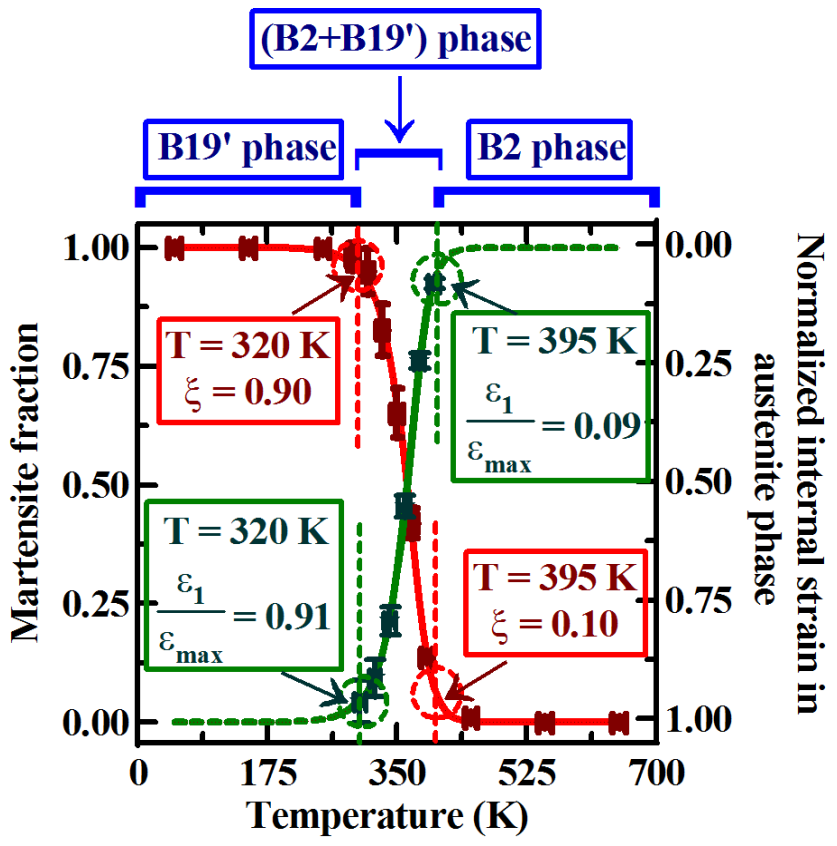

Figure-4: Martensite phase fraction in relation to the internal strain in the austenite and to temperature. The normalized principal strains along [100] or [010] in the tetragonally strained austenite vary linearly with the martensite phase fraction and are described by the same Richards equation as obtained for the evolution of the martensite phase fraction. The phase fractions and 
the strains in the austenite are quantified between the temperature limits corresponding to $90 \%$ and $10 \%$ martensite fraction of the two-phase region. The principal strains are normalized by $\varepsilon_{\max }$, which is the maximum strain in austenite in the microstructure corresponding to $90 \%$ martensite. The $\mathrm{R}^{2}$ values of the optimal fit on martensite fraction and normalized principal strains are 0.9795 and 0.9683 , respectively.

Importantly, Eq.1 also points to the fact that the same Richards equation (with $g=0.04 \pm$ $0.003 \mathrm{~K}^{-1}, \quad v=1.994 \pm 0.019$ and $\quad T_{m}=363.4 \pm 12.1 \mathrm{~K}$ ) accurately describes the principal strains (along the directions parallel to the habit plane) as a function of temperature, when the principal strains are normalized with respect to the strain at $90 \%$ martensite phase fraction ( $\varepsilon_{\max }=2.55 \pm 0.09 i$. However, the strain perpendicular to the habit plane, i.e. along the [001] direction does not obey the same Richards equation (it follows a Gaussian function). Such a correlation between the principal strains and the martensite fraction implies that the strains in austenite parallel to the habit plane are responsible for its stabilization and scale linearly with the martensite fraction. Furthermore, our MD simulation results show a systematic increase ( $\sim 40 \mathrm{~K})$ in the transformation start and finish $\left(\mathrm{M}_{\mathrm{s}}\right.$ and $\left.\mathrm{M}_{\mathrm{f}}\right)$ temperature than those reported in the literature $[13,31]$. This systematic shift can be attributed to the fact that in our study, single crystal supercells were subjected to isothermal equilibration conditions, while in the cited literature, either polycrystalline NiTi samples were considered [31] or the samples were subjected to continuous heating/cooling [13], leading to changes in the transformation temperatures [13,31].

The simulation results (i.e. martensite phase fraction and internal strain in austenite phase) is found to be independent of the orientation of the supercells. To demonstrate this, a simulation cell of B2 with [111] orientation is considered (size 76.5 $\AA \quad 76.5 \AA \quad \times \quad 210 \AA$ ), and the results (i.e. the martensite phase fraction and internal strain in austenite phase) are compared with the [001] oriented B2 cell (75 $\times \quad 75 \AA \quad \times 200 \AA$ ), as shown in Figure 5.
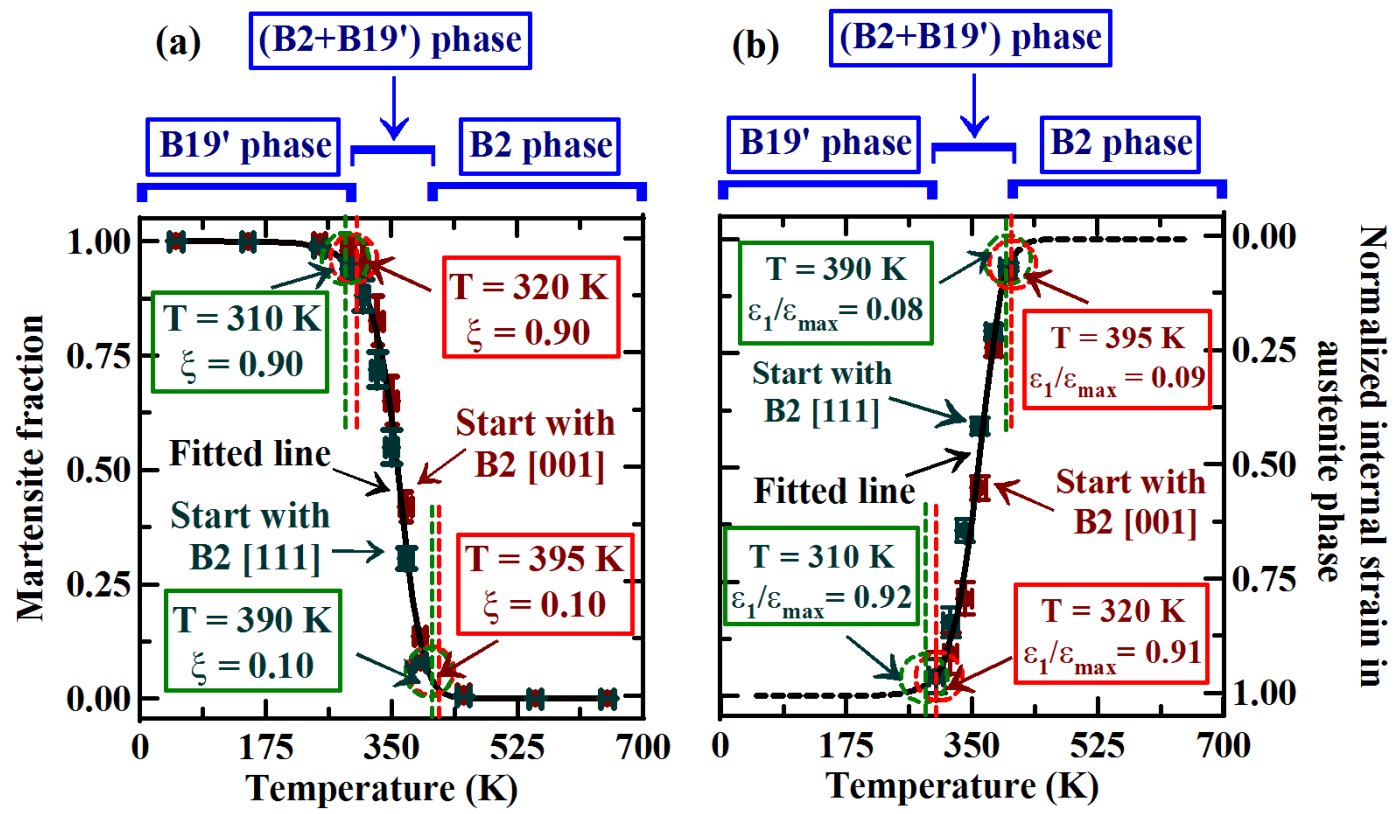
Figure-5: Variation of (a) martensite phase fraction and (b) internal strain in austenite in the twophase region as a function of temperature for supercell size III with different crystal orientation ([001] and [111] B2 crystal). Black line shows the fitted Richards equation for martensite phase fraction and internal strain in austenite phase as a function of temperature.

Figure 5a shows the temperature dependency of the martensite phase fraction, obtained from the [001] and [111] B2 simulation cells. It is clearly observed that the same Richards equation could be used for the temperature dependent evolution of martensite phase fraction. Similarly, Fig. 5b shows the evolution of internal strain in austenite phase with temperature and it can be observed that for both the orientations of B2 crystal, internal strains are similar and follow same Richards' equation.

\subsection{Phonon band structure of austenite phase}

As mentioned earlier the displacive phase transformation of austenite occurs via the phonon instability on the $\mathrm{TA}_{2}$ branch. In order to examine the role of temperature and the developed strain in austenite within the two-phase region to the stability of the $\mathrm{TA}_{2}$ phonon branch, the anharmonic phonon dispersion of the austenite phase as a function of temperature is evaluated. As seen in sections 3.2 and 3.3, while at temperatures greater than the martensite start (in the single phase region) the austenite phase is in its stable cubic structure, at temperatures corresponding to the two-phase region, the austenite phase is characterized by tetragonal symmetry. Thus by employing the stable crystal structures and corresponding lattice parameters depending on temperature, the phonon dispersions of the austenite phase are calculated from $\mathrm{MD}$, primarily focusing on the softening of the $\mathrm{TA}_{2}$ branch. However, the predicted phonon band structures from classical MD potentials, which were parameterized by employing the bulk properties (from experiments and first-principles), may not yield a quantitatively accurate description of phonons as a function of volume and temperature. In other words, since the employed classical MD potentials were not trained against the correct mode Gruneisen parameters and phonon-phonon interactions, sufficient care should be exercised in interpretation of these band structures. Hence, in this work analysis of the calculated phonon band structures in comparison to the first-principles predicted phonon dispersions is restricted to examination of the qualitative behavior of the $\mathrm{TA}_{2}$ phonon branch. 

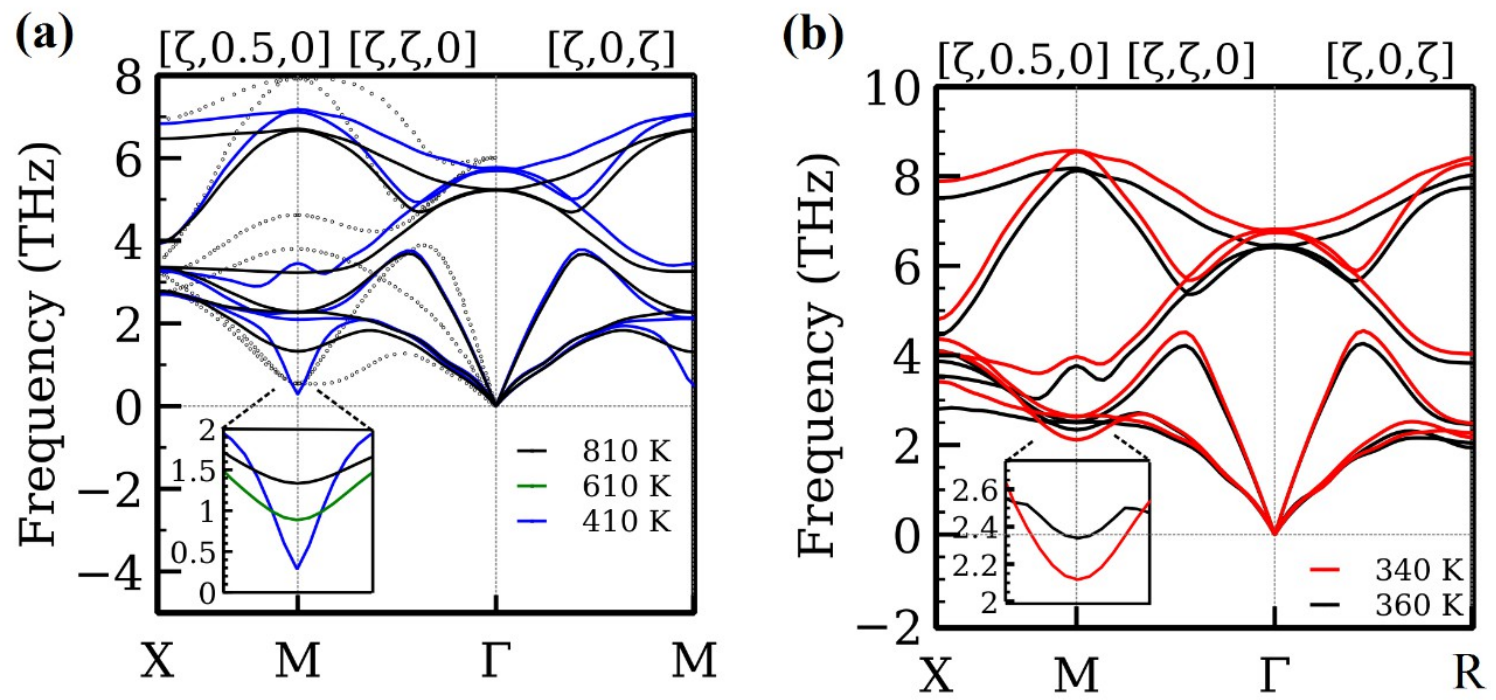

Figure-6: (a) Phonon dispersion of cubic B2 (space group: Pm3m) at different temperatures ( $\mathrm{T}=$ $410 \mathrm{~K}, 610 \mathrm{~K}$ and $810 \mathrm{~K}$ ) within its stable temperature regime illustrating the anomalous softening and onset of instability of the $\mathrm{TA}_{2}$ branch at the zone boundary along the $\Gamma-\mathrm{M}$ direction. The inset shows the $\mathrm{TA}_{2}$ branch at three different temperatures close to the M-point. The circles are the phonon data at $\mathrm{T}=300 \mathrm{~K}$ calculated by first-principles in conjunction with self-consistent ab initio lattice dynamical (SCAILD) method [44] (b) Phonon dispersion of tetragonally strained austenite (Space group: $\mathrm{P} 4 / \mathrm{mmm}$ ) at $\mathrm{T}=340 \mathrm{~K}$ and $360 \mathrm{~K}$ temperature, demonstrating its stabilization in the two-phase region.

Figure 6 shows the phonon dispersion curves of the austenite phase (unstrained and strained) calculated at different temperatures. The cubic B2 structure (space group: Pm-3m) is stable above the $\mathrm{M}_{\mathrm{s}}$ temperature ( $>410 \mathrm{~K}$ ), and the $\mathrm{TA}_{2}$ branch in its phonon dispersion exhibits the known anomalous softening [1,15-17] with decreasing temperature (Fig. 6a). Further, the softening which leads to the instability is observed at the zone boundary along the $\Gamma$-M direction. First-principles investigation of phonon band structures, reported by Souvatzis et al [44], has also reported similar softening behavior of the $\mathrm{TA}_{2}$ branch as a function of temperature. On the other hand, below $\mathrm{M}_{\mathrm{s}}$, the tetragonally strained austenite phase in the two-phase region (Space group: $\mathrm{P} 4 / \mathrm{mmm}$ ) exhibits a stable phonon dispersion (Fig. $6 \mathrm{~b}$ ), as seen by the stable $\mathrm{TA}_{2}$ branch along the $\Gamma-\mathrm{M}$ (and $\Gamma-\mathrm{R}$ ) direction at temperatures corresponding to the two-phase region. Thus, it is safely concluded that below $\mathrm{M}_{\mathrm{s}}$, the countervailing effect of the transformation-induced tetragonal strain is responsible for the stabilization of the austenite phase in the two-phase region. In other words, the resistance for a continued transformation of austenite at a given temperature (between $\mathrm{M}_{\mathrm{s}}$ and $\mathrm{M}_{\mathrm{f}}$ ) is a direct consequence of the tetragonal strain tensor in the austenite phase.

\section{Conclusions}


In summary, molecular dynamics simulations of austenite to martensite phase transformation in NiTi SMA reveal the nature of the transformation-induced strains that stabilize the austenite in the two-phase region between $\mathrm{M}_{\mathrm{s}}$ and $\mathrm{M}_{\mathrm{f}}$. The temperature dependence of the strain tensor in the austenite phase and its effect on the phonon dispersion relations is analyzed. It is found that the principal strains parallel to the habit plane that stabilize the $\mathrm{TA}_{2}$ branch in the phonon dispersion of the austenite phase are found to vary exponentially with temperature in a fashion similar to the martensite phase fraction variation as a function of temperature. The identified similarity between the temperature dependence of the principal strains and the martensite fraction implies that the strains in austenite parallel to the habit plane are responsible for its stabilization and scale linearly with the martensite fraction. Finally, this study provides a new phenomenological model that relates the martensite phase fraction evolution kinetics to the developed internal strains in austenite phase and the temperature.

\section{Acknowledgements}

The reported exploratory work became possible through the support of SG by the University of Arizona. Co-author SB gratefully acknowledges the Thomas G. Chapman Fellowship awarded by the University of Arizona. The authors would like to acknowledge Prof. Ting Zhu (Woodruff school of mechanical engineering at Georgia Institute of Technology) for providing the interatomic potential parameters.

\section{References}

[1] T Tadaki, and K Otsuka, K. Shimizu, Annual Review of Materials Science 18 (1988) 2545.

[2] J. Van Humbeeck, Materials Science and Engineering: A 273-275 (1999) 134-148.

[3] T. Duerig, A. Pelton, D. Stöckel, Materials Science and Engineering: A 273-275 (1999) 149-160.

[4] R.P. Dhote, R.V.N. Melnik, J. Zu, Computational Materials Science 63 (2012) 105-117.

[5] S. Gur, V.R. Manga, S. Bringuier, K. Muralidharan, G. Frantziskonis, in:, 2015 TMS Annual Meeting \& Exhibition, 2015.

[6] S. Gur, G.N. Frantziskonis, Modeling and Simulation in Materials Science and Engineering (2016).

[7] S. Gur, G.N. Frantziskonis, Smart Materials Research (2016).

[8] D. Mutter, P. Nielaba, The European Physical Journal B 84 (2011) 109-113.

[9] J.W. Christian, S. Mahajan, Progress in Materials Science 39 (1995) 1-157. 
[10] T. Waitz, D. Spisak, J. Hafner, H.P. Karnthaler, Europhysics Letters 71 (2005) 98-103.

[11] T. Waitz, W. Pranger, T. Antretter, F.D. Fischer, H.P. Karnthaler, Materials Science and Engineering A 481-482 (2008) 479-483.

[12] T. Waitz, T. Antretter, F.D. Fischer, N.K. Simha, H.P. Karnthaler, Journal of the Mechanics and Physics of Solids 55 (2007) 419-444.

[13] Y. Zhong, K. Gall, T. Zhu, Journal of Applied Physics 110 (2011) 1-9.

[14] Y. Zhong, K. Gall, T. Zhu, Acta Materialia 60 (2012) 6301-6311.

[15] G.L. Zhao, B.N. Harmon, Physical Review B 48 (1993) 2031-2036.

[16] N. Hatcher, O.Y. Kontsevoi, A.J. Freeman, Physical Review B 80 (2009) 144203.

[17] N.A. Zarkevich, D.D. Johnson, Physical Review Letters 113 (2014) 265701.

[18] K. Otsuka, X. Ren, Intermetallics 7 (1999) 511-528.

[19] X. Ren, N. Miura, K. Taniwaki, K. Otsuka, T. Suzuki, K. Tanaka, Y.I. Chumlyakov, M. Asai, Materials Science \& Engineering A (1999) 190 - 194.

[20] K. Otsuka, X. Ren, Progress in Materials Science 50 (2005) 511-678.

[21] Q. Meng, H. Yang, Y. Liu, T. Nam, Intermetallics 18 (2010) 2431-2434.

[22] Y. Chen, C.A. Schuh, Acta Materialia 83 (2015) 431-447.

[23] W. Tirry, D. Schryvers, Nature Materials 8 (2009) 752-7.

[24] S. Qiu, V.B. Krishnan, S.A. Padula, R.D. Noebe, D.W. Brown, B. Clausen, R. Vaidyanathan, Applied Physics Letters 95 (2009) 141906.

[25] K.M. Knowles, D.A. Smith, Acta Metallurgica 29 (1981) 101-110.

[26] K.F. Hane, Journal of the Mechanics and Physics of Solids 47 (1999) 1917-1939.

[27] K.F. Hane, T.W. Shield, Acta Materialia 47 (1999) 2603-2617.

[28] R.D. James, K.F. Hane, Acta Mater. 48 (2000) 197-222.

[29] A.H. Sakhaei, K.-M. Lim, S. Turteltaub, Mechanics of Materials 97 (2016) 1-18.

[30] F. Richards, Journal of Experimental Botany 10 (1959) 290-300. 
[31] N. Zotov, V. Marzynkevitsch, E.J. Mittemeijer, Journal of Alloys and Compounds 616 (2014) 385-393.

[32] S. Plimpton, Computational Materials Science 4 (1995) 361-364.

[33] S. Plimpton, Journal of Computational Physics 117 (1995) 1-19.

[34] D. Mutter, P. Nielaba, Physical Review B 82 (2010) 224201.

[35] D. Mutter, P. Nielaba, Journal of Alloys and Compounds 577 (2013) S83-S87.

[36] Z. Zhang, X. Ding, J. Deng, J. Cui, J. Sun, T. Suzuki, K. Otsuka, X. Ren, The Journal of Physical Chemistry C 117 (2013) 7895-7901.

[37] R. Mirzaeifar, K. Gall, T. Zhu, A. Yavari, R. Desroches, Journal of Applied Physics 115 (2014) 1-8.

[38] T. Sato, K. Saitoh, N. Shinke, Modelling and Simulation in Materials Science and Engineering 14 (2006) S39-S46.

[39] W.S. Lai, B.X. Liu, Journal of Physics: Condensed Matter 12 (2000) L53-L60.

[40] S. Miyazaki, K. Otsuka, Metallurgical Transactions A 17 (1986) 53-63.

[41] M.F.X. Wagner, W. Windl, Acta Materialia 56 (2008) 6232-6245.

[42] O. Mercier, K.N. Melton, G. Gremaud, J. Hägi, Journal of Applied Physics 51 (1980) 1833-1834.

[43] T.M. Brill, S. Mittelbach, W. Assmus, M. Mullner, B. Luthi, Journal of Physics: Condensed Matter 3 (1999) 9621-9627.

[44] P. Souvatzis, D. Legut, O. Eriksson, M.I. Katsnelson, Physical Review B 81 (2010) 092201.

[45] K. Nurveren, A. Akdoğan, W.M. Huang, Journal of Materials Processing Technology 196 (2008) 129-134.

[46] Y. Motemani, M. Nili-Ahmadabadi, M.J. Tan, M. Bornapour, S. Rayagan, Journal of Alloys and Compounds 469 (2009) 164-168. 